\title{
Das Völkerkundemuseum der Universität Zürich 1889-1989
}

\begin{abstract}
Als Ergänzung zur ausführlichen Darstellung der hundertjährigen Geschichte der Geographisch-Ethnographischen Gesellschaft Zürich, welcher Peter Jud im vorangehenden Bericht in differenzierter Weise nachgegangen ist, soll an dieser Stelle ein kurzer Abriß über die Anfänge und die Entwicklung der ehemaligen Ethnographischen Sammlung, dem heutigen Völkerkundemuseum der Universität Zürich, erfolgen. Diese Sammlung war die Gründungsursache und gleichzeitig das Produkt der Ethnographischen Gesellschaft in Zürich, die elf Jahre nach ihrer Gründung gemeinsam mit der etwas jüngeren Geographischen Gesellschaft in der GEGZ aufging.
\end{abstract}

Blenden wir kurz auf die Situation der ethnologischen Forschung im letzten Viertel des 19. Jahrhunderts zurück. Das 19. Jahrhundert brachte sowohl in den Natur- wie in den Geisteswissenschaften rasante Fortschritte, und die Ethnologie, bis dahin lediglich als Hilfswissenschaft und Lieferantin kulturvergleichender Beispiele für die Untermauerung zahlreicher Theorien betrachtet, vermochte sich langsam einen eigenständigen Platz zu erobern. Eigentliche akademische Einrichtungen in diesem Fachbereich begannen sich aber erst gegen Ende des Jahrhunderts zu etablieren. Nicht zu unterschätzen ist bei dieser Entwicklung der sich beschleunigende Wettlauf der Kolonialmächte im Kampf um die letzten noch verbliebenen «weißen Flecken» der Welt, deren Eroberung wirtschaftliche Prosperität und politische Macht zu versprechen schienen. Nicht selten wurden Wissenschafter in den neu eroberten Gebieten eingesetzt, die mit ihren ethnographischen Forschungen Strukturen aufdecken sollten, durch deren Kenntnis die Hegemonialmacht das Gebiet zu regieren und zu halten hoffte. Gewiß sind diesbezüglich bei den einzelnen Kolonialmächten große Unterschiede festzustellen, so war es vor allem eine französische Spezialität, Ethnologen in der oben genannten Weise einzusetzen, die Engländer hingegen mit ihrem Prinzip des «indirect rule» machten davon deutlich weniger $\mathrm{Ge}$ brauch.

Wo stand nun aber die Schweiz? Sie konnte sich zu keiner Zeit Chancen auf politische Eroberung von Kolonialgebieten ausrechnen. Vorausschauende Wirtschaftskreise sahen hingegen schon frühzeitig, daß sie versuchen mußten, sich wenigstens einen
Anteil an den zu erwartenden wirtschaftlichen Erfolgen zu sichern. In diesem Zusammenhang ist auch das Engagement zu sehen, mit dem die Schweiz, vertreten durch ein Komitee der Geographischen Gesellschaft in Genf, die Idee des Königs Leopold II. von Belgien vehement unterstützte, Zentralafrika und insbesondere das Kongogebiet zu erobern und zu erschließen.

Der Zoologe Conrad Keller (1848-1930), unter dessen Vorsitz am 23. Februar 1888 die Ethnographische Gesellschaft zum ersten Mal zusammentrat, hatte seine ersten Erfahrungen in den vielfältigen Aspekten der Erschließung von außereuropäischen Gebieten in der 1878 in St. Gallen gegründeten «Ostschweizerischen Geographisch-commerciellen Gesellschaft» gewinnen können. Zusammen mit dem Ethnologen Otto Stoll (1849-1922) und dem Kaufmann Fritz Rieter ließ er sich durch diese auf kommerzielle Belange ausgerichtete Gesellschaft zur Gründung einer ethnographischen Vereinigung in Zürich inspirieren, die trotz offen dargelegter Wirtschaftsfreundlichkeit darüber hinaus ideelle Ziele anstreben sollte. Im vorbereitenden "Circular betreffend Gründung einer ethnographischen Gesellschaft» von 1887 waren Zweck und Aufgabe bereits mit folgendem Wortlaut ausformuliert: "Die Ethnographische Gesellschaft in Zürich stellt sich die Aufgabe, das Gesammtgebiet der Völkerkunde in theoretischer und praktischer Hinsicht zu fördern. Um diesen Zweck zu erreichen, übernimmt sie in erster $L i$ nie die Schöpfung, Unterhaltung und Mehrung eines ethnographischen Museums und betrachtet sich bis auf weiteres vorwiegend als Garantiegesellschaft zur Unterhaltung des zu gründenden Museums.» 17 Persönlichkeiten, zur Hauptsache Professoren, Wirtschaftsleute und Politiker, die im Bericht von Peter Jud namentlich aufgeführt sind, dokumentierten mit ihrer Unterschrift die tatkräftige Unterstützung der neuen und für Zürich neuartigen Gesellschaft. Ein weiterer Programmpunkt im erwähnten Circular be-

Verena Münzer, lic. phil. I, Ethnologin, Resedastr. 20, 8008 Zürich 
zog sich auf die für unverzichtbar gehaltene praktische Anwendbarkeit der Völkerkunde: «Da hochentwickelte Kulturnationen auf zahlreichen Handelswegen Fühlung mit tieferstehenden Kulturvölkern suchen, um diese wirthschaftlich dienstbar zu machen, so hat die Pflege der Ethnologie neben ihrem geistig bildenden Wert auch eine eminent practische Bedeutung gewonnen.» Mit diesen Worten wird gleichzeitig eine allgemein evolutionistische Sichtweise zum Ausdruck gebracht, welche in der Zeit von 1860 bis 1900 die wissenschaftlichen Diskussionen und Theorien im Bereich der Ethnologie vorwiegend prägte. Gemäß ihrer Hauptaufgabe schuf die Ethnographische Gesellschaft schon am Anfang neben den vereinsüblichen Ämtern den Posten eines Sammlungsdirektors, welchen sie mit Otto Stoll, einem gelernten Mediziner und autodidakten Ethnologen, für die ersten elf wichtigen Aufbaujahre besetzte. Es sei an dieser Stelle erlaubt, kurz auf die wichtigsten Lebensstationen dieses für die Entwicklung der Ethnologie in Zürich wichtigen Vertreters einzugehen. Otto Stoll wandte sich nach einem längeren Aufenthalt in Guatemala (1878-1883) von seinem Beruf als Arzt ab und habilitierte sich in der Folge 1884 mit einer Arbeit «Zur Ethnographie der Republik Guatemala» an der damaligen philosophischphilologisch-historischen Sektion der Philosophischen Fakultät der Universität Zürich zum Privatdozenten für Ethnographie und Anthropologie. Gleichzeitig hatte er auch eine Lehrverpflichtung (1886-1895) am Polytechnikum Zürich. Dieser Werdegang ist typisch für die meisten Professoren der ersten Generation, welche die frühen ethnologischen Lehrstühle besetzten. Zahlreiche Pioniere dieses Wissenschaftsbereiches waren sogenannte Amateurgelehrte und rekrutierten sich aus dem naturwissenschaftlichen Umfeld, vor allem aus Anthropologie, Medizin, Zoologie und Geographie. So war auch der berühmte Adolf Bastian, der zur zentralen Figur bei der Institutionalisierung der Ethnologie an deutschen Universitäten wurde, indem er sich um den Aufbau von großen völkerkundlichen Sammlungen kümmerte, ursprünglich Schiffsarzt gewesen. Durch seine vielen Reisen angeregt, wandte er sich schon früh ganz der ethnologischen und ethnographischen Forschung zu. Otto Stoll las die zahlreichen - nicht immer leicht verständlichen - Publikationen Bastians mit großem Interesse und allgemeiner Zustimmung, welcher er mit einer Widmung in seiner 1889 publizierten Arbeit über «Die Ethnologie der Indianerstämme von Guatemala» Ausdruck verlieh. Die weiteren beruflichen Stationen führten Otto Stoll vom Extraordinariat (1891/92) zum Ordinariat (1895) für Geographie, das ab diesem Zeitpunkt der mathematischnaturwissenschaftlichen Sektion angegliedert war. Nach den wenigen zaghaften Versuchen in den Anfangsjahren der Universität Zürich und dem kurzen Gastspiel Wilhelm Wundts 1874/75 fanden jetzt erst- mals regelmäßig Vorlesungen in einem breiten ethnologischen Spektrum statt.

Nach etwas mehr als einem Jahr an Vorbereitungszeit wurde an der Hauptversammlung der Ethnographischen Gesellschaft vom 23. Mai 1889 bekanntgegeben, daß nun die Sammlung jeweils am Mittwochnachmittag für ein allgemeines Publikum geöffnet sei. Dieses Datum bildet den eigentlichen Auftakt für die hundertjährige Geschichte des heutigen Völkerkundemuseums. Die Sammlungen, die damals die Basis der nach geographischen Gesichtspunkten gegliederten Ausstellung bildeten und im Kuppelraum der alten Börse gezeigt wurden, hatte die Gesellschaft im Laufe des Jahres 1888 zusammengetragen. Es handelte sich dabei um ziemlich zufällige, eher kleine und oft nur aus Alltagsgegenständen bestehende Objektgruppen. Oft waren die Sammler selbst Mitglieder der Gesellschaft und kamen mit Schenkungen oder auch Verkäufen den Forderungen nach tatkräftiger Unterstützung des neuen $\mathrm{Mu}$ seums nach. Dabei legte die Ethnographische Gesellschaft immer das größte Gewicht auf das von Anfang an verfolgte Prinzip, die Sammlung solle in erster Linie allen Unterrichtsstufen sowie den jungen Kaufleuten zur Anschauung und Belehrung dienen.

Nach dieser Aufbauphase, die in der Eröffnung der Ethnographischen Sammlung ein erstes Ziel erreichte, waren die folgenden Jahre in erster Linie von Finanz- und Raumproblemen geprägt. Interessante Sammlungen konnten oft nur dank der privaten Spenden einzelner Gesellschaftsmitglieder gekauft werden, wenn die allgemeinen Mitgliederbeiträge dazu nicht ausreichten.

1894 übersiedelte die Ethnographische Sammlung von der alten Börse, welche die kaufmännische Gesellschaft in großzügiger Weise zur Verfügung gestellt hatte, in ein Haus am Seilergraben. Hoffnungen, die Sammlung einst in den Räumen des in diesen Jahren geplanten Landesmuseums unterzubringen, waren definitiv gescheitert. Diese Erfahrungen brachten die Ethnographische Gesellschaft schon 1898 dazu, die Sammlung, welche zu einer immer größeren Belastung für die kleine Gesellschaft wurde, dem Kanton Zürich zu schenken, der sich gerade damit befaßte, das Bauprogramm für das neue Universitätsgebäude zu entwerfen. Einzige Bedingung von seiten der Gesellschaft war, daß der Charakter einer Unterrichtssammlung gewahrt bleiben sollte. Die Verhandlungen waren schließlich erfolgreich und führten dazu, daß schon im Bauprogramm Räume für die Ethnographische Sammlung eingeplant wurden.

Otto Stoll überließ Ende 1898 das Amt des Sammlungsdirektors Rudolf Martin (1864-1925), der sich 1891 an der Universität und am Polytechnikum Zürich als Privatdozent für physische Anthropologie habilitiert hatte. 1899 erfolgte schließlich die Grün- 


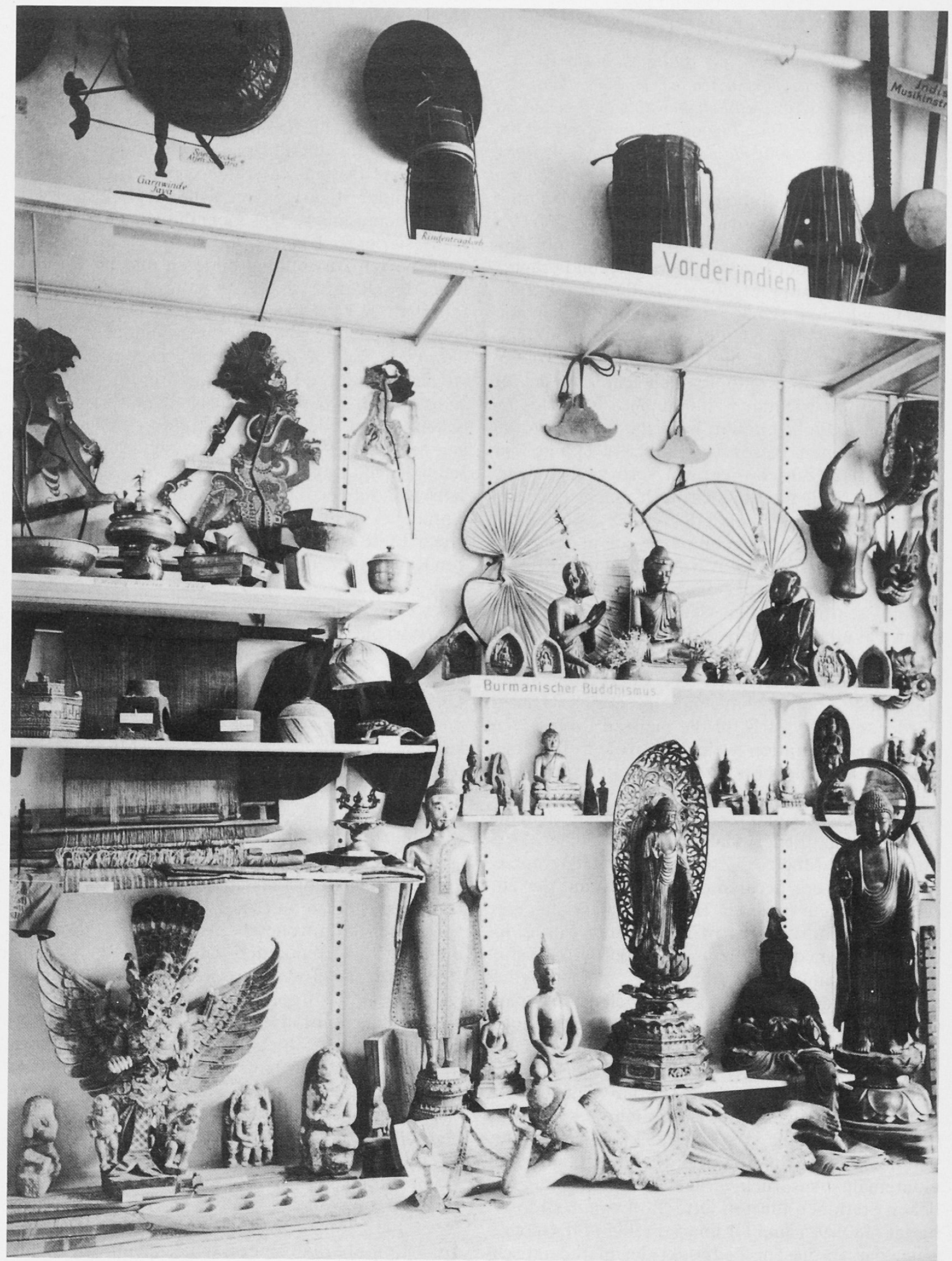

Abb. 1 Die Sammlung für Völkerkunde der Universität Zürich im Ausstellungsbereich Asien, wie sie sich um 1920 unter der Leitung Hans Wehrlis präsentierte.

(Foto: Völkerkundemuseum der Universität Zürich) 


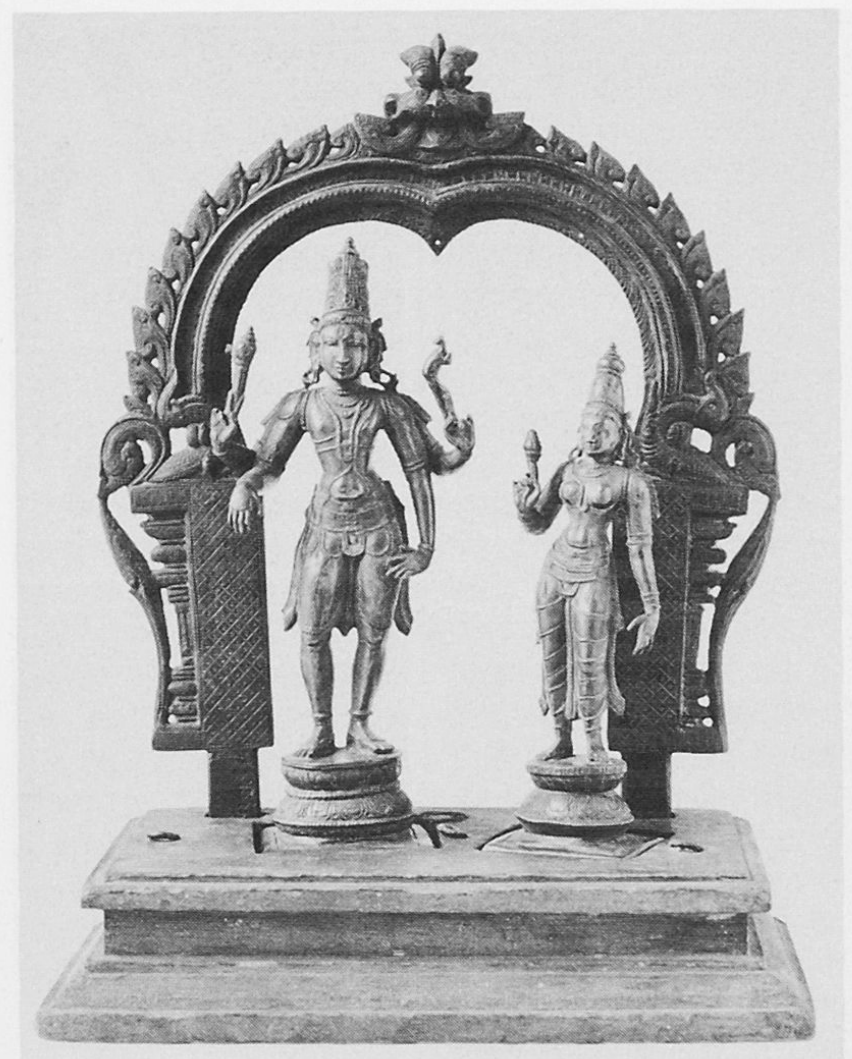

Abb. 2 Bronzefiguren Shiva und Parvati (Indien), welche Hans Wehrli von einer 1926/27 zusammen mit Martin Hürlimann durchgeführten Reise zurückbrachte.

(Foto: Peter Nebel, Völkerkundemuseum der Universität Zürich)

dung eines Lehrstuhles für physische Anthropologie an der Universität Zürich, nachdem Rudolf Martin durch seine Arbeit konsequent auf dieses Ziel hingearbeitet hatte. Die Wahl eines Anthropologen zur Betreuung der Ethnographischen Sammlung macht deutlich, daß zu jenem Zeitpunkt Anthropologie und Ethnologie noch eine Art Konglomerat bildeten. In seiner Antrittsrede, 1900 an der Universität Zürich gehalten, nimmt Rudolf Martin selbst eine Abgrenzung der beiden Wissenschaften vor: «Meiner Ansicht nach nun zerfällt die „Anthropologie im weiteren Sinne" naturgemäß in zwei, durch das $\mathrm{Ob}$ ject eng verbundene Wissenschaften: in die Physische Anthropologie, auch Morphologie, oder Somatologie der Menschenrassen genannt und in die Psychische Anthropologie, die auch unter der Bezeichnung Ethnologie oder Völkerkunde bekannt ist.»

Als Rudolf Martin das Amt der Sammlungsbetreuung übernahm, brachte der nur wenig später stattfindende Zusammenschluß der beiden bis dahin unabhängigen Gesellschaften für Ethnographie und Geographie eine wesentlich veränderte Situation. Obwohl die beiden Fachrichtungen weiterhin - und jetzt unter einer Führung vereint - gepflegt werden sollten und die Verantwortung für die Sammlung ebenfalls an die neue Gesellschaft überging, verlagerten sich die Interessen und Aktivitäten zusehends auf die Seite der Geographie hin.

1909 - die Sammlung war in den vergangenen zehn Jahren nicht mehr in gleichem Ausmaß gewachsen wie in der Anfangszeit - wurde Hans J. Wehrli (1871-1945) Nachfolger von Rudolf Martin in der Betreuung der Ethnographischen Sammlung. Hans Wehrli, der auch als Präsident der GeographischEthnographischen Gesellschaft während Jahrzehnten eine große Rolle spielen wird, trat 1913 die Nachfolge Otto Stolls als Ordinarius für Geographie mit Einschluß von Völkerkunde und Wirtschaftsgeographie an. Die Ethnographische Sammlung wurde im gleichen Jahr, nach langwierigen Verhandlungen, definitiv vom Kanton Zürich übernommen - notabene als Geschenk - und der neuen Universität angegliedert. Nachdem die neuen Bestimmungen des Kantons Zürich den jeweiligen Lehrstuhlinhaber im Bereich Völkerkunde als Sammlungsdirektor vorsahen, verblieb diese Aufgabe weiterhin bei Hans Wehrli.

Die Tätigkeit in der Sammlung, die völlig neu konzipiert werden mußte, wurde mehr denn je in den nun folgenden Jahren allein von der Person des Direktors bestimmt, weil keine Sammlungsgesellschaft mehr im Hintergrund wirkte. Nach der Einweihung des neuen Universitätsgebäudes im Frühjahr 1914 dauerte es über zwei Jahre, bis die neugestaltete «Sammlung für Völkerkunde der Universität Zürich» am 13. Dezember 1916 ihre Pforten öffnete. Wissenschaftlich stellte die Gestaltung der Sammlung Hans Wehrli vor einige Schwierigkeiten, zumal man nach damaliger Auffassung die ganzen Sammlungsbestände permanent in der Ausstellung zeigen wollte und insgesamt enzyklopädische Vollständigkeit anstrebte. Ziel war dabei die Herausarbeitung einzelner Kulturstufen der Menschheit, deren Einteilung meistens nur den Grad der technologischen Beherrschung berücksichtigten. Hans Wehrli ergänzte die traditionelle Schau mit kleinen vergleichend aufgebauten Ausstellungsteilen, wobei er zum Beispiel Tausch- und Zahlungsmittel zeigte. Mit Hilfe der vergleichenden Methode versuchte man Ähnlichkeiten und Divergenzen zu erkennen, die zu allgemeinen Aussagen über Ursprung und Entwicklung der Menschheit führen sollten.

Die finanzielle Situation der Sammlung ist während dieser Jahre bescheiden. Kanton und Stadt Zürich und auch die GEGZ, welche die Förderung der Sammlung weiterhin in ihren Statuten verankert hat, beteiligten sich regelmäßig mit kleinen Beiträgen, die für Ankäufe von Ethnographica verwendet wurden. 1917 wird mit dem Anlegen einer Handbibliothek begonnen, die zwar schon von Otto Stoll 1895 angeregt, aber nie in die Tat umgesetzt worden war. 


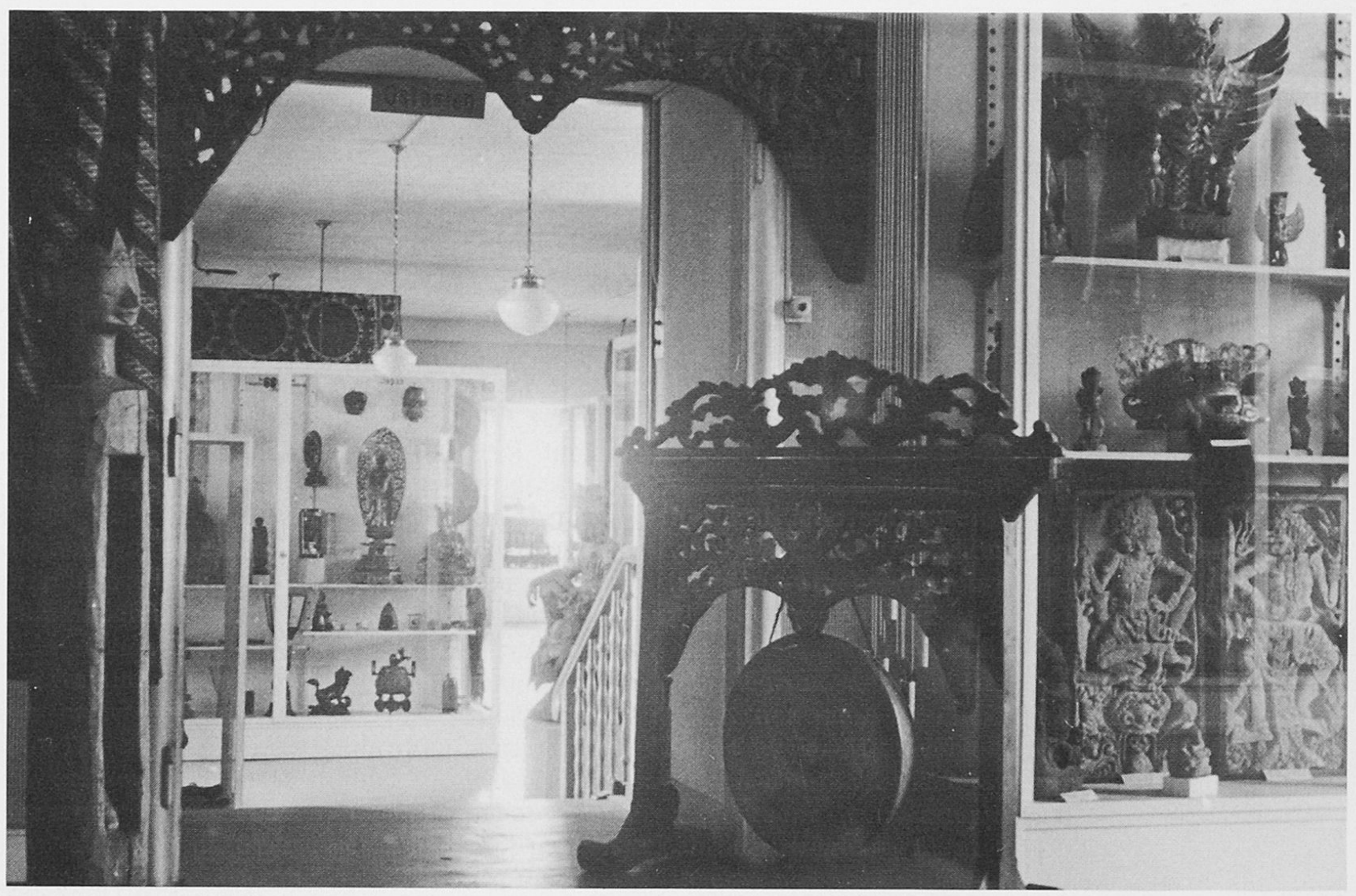

Abb. 3 Die Sammlung in den vierziger Jahren in der Gestaltung von Alfred Steinmann.

(Foto: Völkerkundemuseum der Universität Zürich)

Der Rücktritt Hans Wehrlis als Ordinarius für Geographie brachte eine Aufspaltung der bisher zusammengelegten Fachbereiche Geographie und Ethnologie mit sich: Hans Boesch übernahm 1942 den Lehrstuhl für Geographie und die Leitung des Geographischen Instituts, während die Völkerkunde im Rahmen der Geographie ab 1939 vom Privatdozenten Alfred Steinmann (1892-1974) betreut wurde. Alfred Steinmanns Hauptaufgabe lag jedoch in der Weiterführung der Sammlung für Völkerkunde, die 1942 in administrativer und finanzieller Hinsicht institutionell vom Geographischen Institut getrennt wurde. Zu diesem Zeitpunkt konnte Alfred Steinmann bereits auf eine lange Berufslaufbahn als Botaniker zurückblicken. Insgesamt siebzehn Jahre hatte er in holländischen Diensten in Buitenzorg auf Java verbracht und dort in Versuchsstationen für Kautschuk, Tee und Kakao gearbeitet. Diese reiche Felderfahrung in Indonesien führte ihn schließlich 1937 zum Studium der Ethnologie, vorerst in Wien und anschließend in Zürich, wo er sich 1939 mit der Arbeit «Das kultische Schiff in Indonesien» bei Hans Wehrli habilitierte. Als Sammlungsdirektor zeichnete er während seiner mehr als zwanzigjährigen Amtszeit für zahlreiche Umgruppierungen der
Ethnographica verantwortlich, ohne allerdings das alte Ausstellungsprinzip im wesentlichen anzutasten, das er 1956 mit folgenden Worten umriß: «Obschon die Anordnung der Bestände im Prinzip nach geographischen Gesichtspunkten erfolgte, wurde im einzelnen stets danach getrachtet, innerhalb der einzelnen Gebiete die Entwicklung von primitiven zu höheren Kulturformen möglichst anschaulich zur Darstellung zu bringen.» Diese evolutionistischen Ansätze galten jedoch in den meisten ethnologischen Kreisen Ende der fünfziger Jahre als veraltet.

Mit der Ablösung Alfred Steinmanns als Direktor 1963 durch den Religions- und Kunstethnologen Karl H. Henking, der bis heute dem Museum vorsteht, bahnte sich zugleich eine neue Aera für die völkerkundliche Sammlung an - weg vom statischen und hin zu einem dynamischen Prinzip der Ausstellungsgestaltung. Diese neue Dynamik war nur möglich, indem sich Karl Henking intensiv um personellen, finanziellen, strukturellen und räumlichen Aufund Ausbau bemühte. Um die neuen Konzepte realisieren zu können, war die Sammlung von 1968 bis 1971 öffentlich nicht mehr zugänglich. Während dieser Zeit wurden auch die Räumlichkeiten einer gründlichen Renovierung unterzogen, die dringend 
benötigte Vergrößerung der Ausstellungsfläche konnte jedoch auch jetzt nicht gewährt werden. Die insgesamt erfreuliche Entwicklung der Sammlung für Völkerkunde wurde ohne Zweifel indirekt durch den gesamtschweizerischen Trend unterstützt, der sich in der Erstarkung und Verselbständigung der Ethnologie als einer von der Geographie unabhängigen Disziplin manifestierte. Zwei Ereignisse wurden 1971 sowohl für die Sammlung wie für das Fach Ethnologie an der Universität Zürich richtungsweisend: im Juni wird die «Schweizerische Ethnologische Gesellschaft» (SEG) gegründet, und wenig später wird Lorenz G. Löffler als neuer Ordinarius an die Philosophische Fakultät I berufen. Damit wurde die Ethnologie zu einem unabhängigen Fach, welches gleichzeitig aus der naturwissenschaftlich ausgerichteten Fakultät in den geisteswissenschaftlichen Rahmen wechselte. Im Oktober 1971 löste sich zudem offiziell die noch lose bestehende Verbindung der Sammlung zur Geographie auf. Indem die Sammlung als «Völkerkundemuseum der Uni-

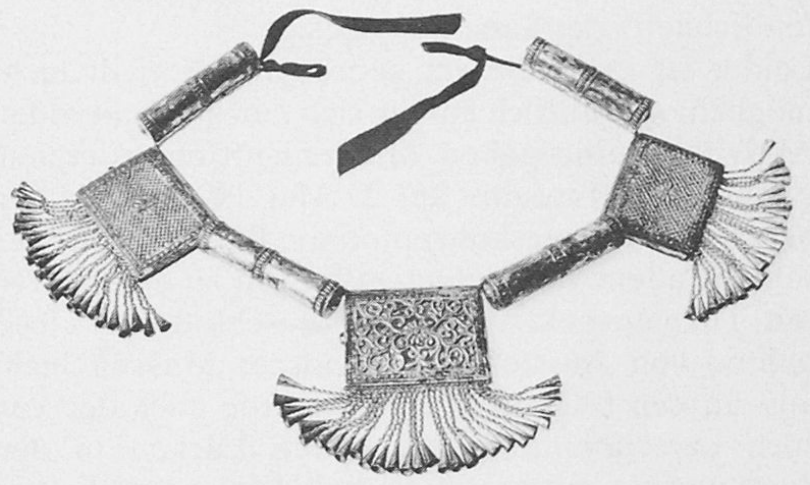

Abb. 4 Amulettkette aus der Sammlung IIg (Äthiopien). Alfred Ilg, an verschiedenen Bauprojekten in Äthiopien beteiligt, gelangte als Staatsminister unter Kaiser Menelik II zu einiger Berühmtheit.

(Foto: Peter Nebel, Völkerkundemuseum der Universität Zürich)

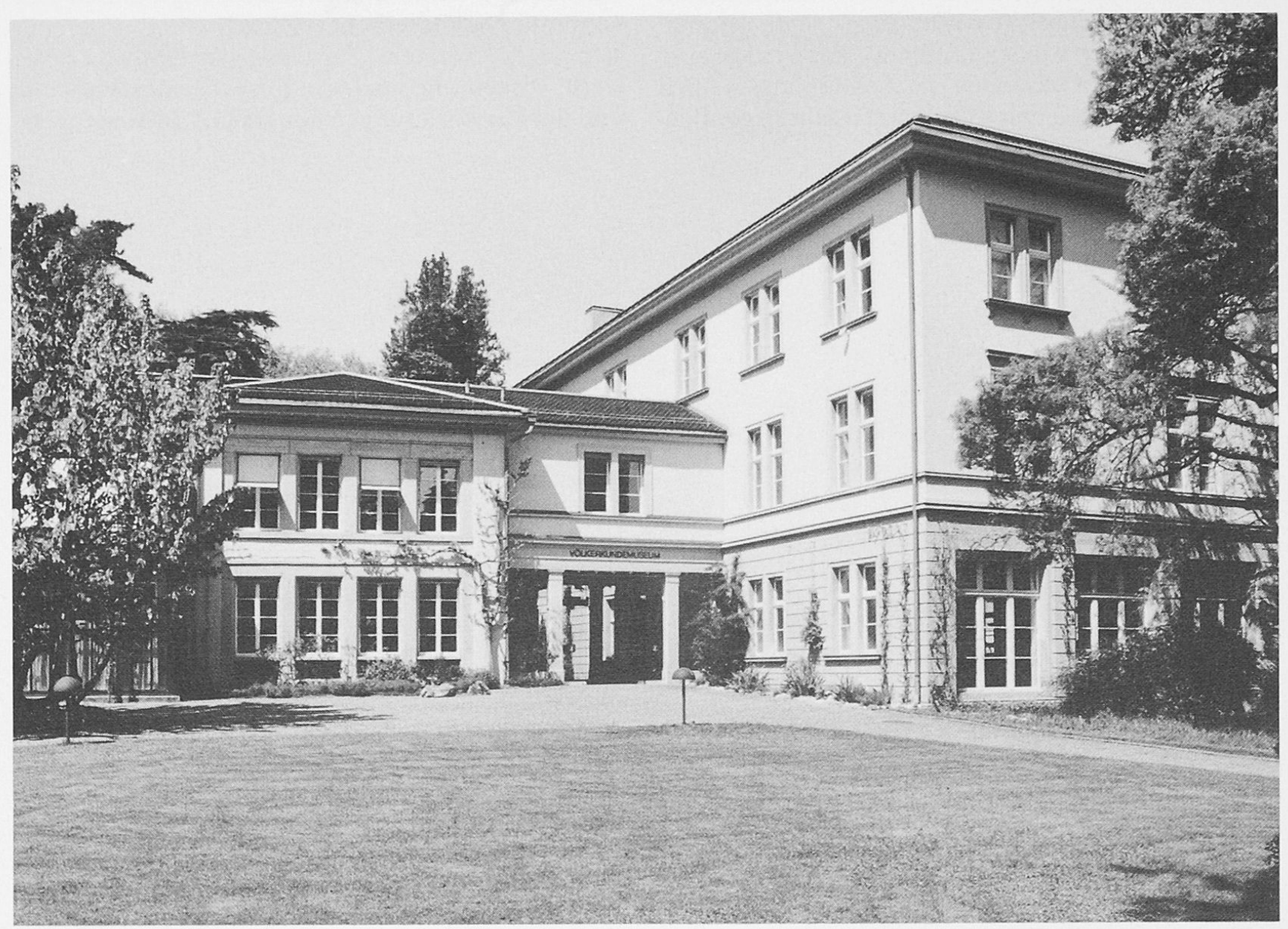

Abb. 5 Das Völkerkundemuseum der Universität Zürich heute im Areal des alten Bollwerkes "Zur Katz", im ehemaligen Botanischen Garten.

(Foto: Julia Hintermüller, Völkerkundemuseum der Universität Zürich) 
versität Zürich» ihren bis heute gültigen Namen erhielt, wurde die Betonung vermehrt auf die «Öffentlichkeit» der Sammlung gelegt.

Leider ist es in diesem gedrängten Bericht nicht möglich, ausführlich auf die sich nun auffächernden Aktivitäten einzugehen. Mit der Eröffnung des neugestalteten Museums am 2. Mai 1972 wurde ein neues Ausstellungskonzept in die Wege geleitet: Es sah vor allem Wechselausstellungen zu einem breiten Themenspektrum vor und schloß die Übernahme von Ausstellungen anderer Museen nicht aus. In den folgenden Jahren wurde auch der Versuch unternommen, bestehende Lücken in den Sammlungen mit gezielten Ankäufen zu schließen. Obwohl nach dem neuen Ausstellungsprinzip nicht mehr das ganze Sammlungsgut präsentiert werden mußte, nahmen die Platzprobleme schon bald wieder überhand. Die für 1974 geplante Verlegung der Botanischen Institute an die Zollikerstraße bot die Möglichkeit, die Gebäude im alten Botanischen Garten für das Völkerkundemuseum umzubauen. Diesem Vorhaben stimmte der Kantonsrat im Dezember 1976 zu. Im Spätsommer 1979 erfolgte dann der Umzug des Museums.

Als am 31. Oktober 1980 das Völkerkundemuseum im Areal des Bollwerkes «Zur Katz» seine öffentlichen Aktivitäten wieder aufnimmt, kann es mit einigen Neuerungen besonders im Ausstellungsbereich aufwarten. Zu den erst seit wenigen Jahren gepfleg- ten Wechselausstellungen und den althergebrachten längerfristigen sogenannten Dauerausstellungen, in denen vor allem die Sammlungsbestände zugänglich gemacht werden sollen, kommt neu die «Studiensammlung» hinzu. Mit dieser Maßnahme wollte man wieder dem alten Grundsatz der Sammlung, hauptsächlich der Lehre zu dienen, trotz den vielfältiger gewordenen Anforderungen nachkommen.

Dem Museum ist mit dem neuen Standort die oft formulierte Aufgabe aufgetragen worden, als eines der «Schaufenster der Universität» zu wirken, was sich in den verstärkten Bemühungen widerspiegelt, wissenschaftlich erarbeitete Erkenntnisse aus dem weiten Bereich der Ethnologie in gut verständlicher Form einem vielseitig interessierten Publikum zu präsentieren. Dabei spielen Ausstellungen nicht mehr die alleinige Rolle der Wissensvermittlung. Ein zunehmendes Angebot von Veranstaltungen Vorträge, Tagungen, Dokumentarfilme, Tanz- und Musikaufführungen, Kurse für Kinder und Erwachsene - locken immer mehr Besucher ins Museum. Das Bemühen um Ganzheitlichkeit und der interdisziplinäre Charakter sowohl der Ethnologie wie auch der Geographie sind nach wie vor verbindende Elemente. Das Museum und die Geographisch-Ethnographische Gesellschaft Zürich könnten gemeinsam wieder vermehrt für diese ganzheitliche Sichtweise wirken und auch die Besucher des Museums und der Veranstaltungen der GEGZ dazu anregen. 


\section{Quellen}

AEPPLI, A. (1923): "Jakob Früh zu seinem siebzigsten Geburtstag am 22. Juni 1922." In: Mitteilungen der Geographisch-Ethnographischen Gesellschaft Zürich, Bd. XXII (1921/22).

AMREIN, K. C. und KÜNZLE-STEGER (1879): «Bericht über die Verhandlungen des Congrès International de Géographie Commerciale de Bruxelles vom 27. September bis 1. Oktober 1879." In: Jahresbericht der Ostschweizerischen Geographisch-commerciellen Gesellschaft pro 1879. St. Gallen.

BECK, H. (1982): Große Geographen. Pioniere-Außenseiter - Gelehrte. Berlin: Dietrich Reimer.

BECKER, F. (1897): "Das topographische Relief in seiner Bedeutung für die Landeskunde." In: Schweizerische Monatschrift für Offiziere aller Waffen, 9. Jg. (1897).

BOESCH, H. (1946): "Geographie." In: Festschrift zur 200Jahr-Feier der Naturforschenden Gesellschaft in Zürich. Zürich: Fretz.

BOESCH, H. (1962): "Zur Stellung der modernen Geographie.» In: Geographica Helvetica, Bd. XVII (1962).

BRUNNER, H. R. (1980): "Geographisches Institut.» In: Eidgenössische Technische Hochschule Zürich, 1955-1980. Festschrift zum 125jährigen Bestehen, hg. vom Rektor der ETH Zürich und red. von Jean-François Bergier und Hans Werner Tobler. Zürich: Neue Zürcher Zeitung.

BÜHLER, A. (1972): "Die Aufgaben des Ethnologen.» In: Ethnologische Zeitschrift Zürich, II (1972).

DE CLAPARĖDE, A. (1908): Coup d'œil sur la Société de Géographie de Genève depuis sa fondation en 1858. Genf: Atar.

DÜRST, A. (1980): «Professor Dr. Fritz Müller (16. April 1926-26. Juli 1980)." In: Geographica Helvetica, 35. Jg (1980), Nr. 3.

EGLI, E. (1961): "Die Geographie in Wissenschaft und Bildung." In: Mensch und Landschaft. Kulturgeographische Aufsätze und Reden. Zürich: Artemis, 1975.

EGLI, E. (1964): «Rückblick auf die jüngsten 25 Jahre der Geographisch-Ethnographischen Gesellschaft Zürich, 1939-1964." In: Geographica Helvetica, Bd. XIX (1964), Nr. 3.

FISCHER, H. (1988): Ethnologie. Einführung und Überblick. Berlin: Reimer.

FRÖBEL, J. (1890-91): Ein Lebenslauf. Aufzeichnungen, Erinnerungen und Bekenntnisse. 2 Bde. Stuttgart: Cotta'sche Buchhandlung.

FURRER, G. (1981): "Karl Suter (1901-1981). In: "Geographica Helvetica, 36. Jg. (1981), Nr. 2.

GAGLIARDI, E. und STROHL, J. (1938): «Die Universität Zürich 1833-1933." In: Die Universität Zürich 1833-1933 und ihre Vorläufer. Festschrift zur Jahrhundertfeier, hg. vom Erziehungsrate des Kantons Zürich, bearb. von Ernst Gagliardi, Hans Nabholz und Jean Strohl. Zürich: Erziehungsdirektion.

Geographische Zeitschriften (div. Jahrgänge):

Bulletin de la Société de Géographie de Genève/Le Globe. Jahresberichte der Geographischen Gesellschaft in Bern. Mitteilungen der Ostschweizerischen Geographisch-commerciellen Gesellschaft.

Bulletin de la Société neuchâteloise de Géographie.

Jahresberichte der GEGZ/Mitteilungen der GEGZ/Geographica Helvetica.

Mitteilungen der Geographisch-Ethnographischen Gesellschaft in Basel.
GUTERSOHN, H. (1942): "Otto Flückiger (1881-1942)." In: Mitteilungen der Geographisch-Ethnographischen Gesellschaft Zürich, Bd. XXXXI (1941/42 und 1942/43).

GUTERSOHN, H. (1946): "Geographica Helvetica." In: Geographica Helvetica, 1. Jg. (1946), Nr. 1.

HEIM, A. (1944): "Die naturwissenschaftlichen Arbeiten von Arnold Heim 1905-1943 (Autoreferat)." In: Vierteljahrsschrift der Naturforschenden Gesellschaft in Zürich, 89. Jg. (30. Sept. 1944), Beiheft Nr. 3.

HENKING, K. H. (1980): Das Völkerkundemuseum der Universität Zürich. Geschichte und Ausblick. Zürich.

IMHOF, E. (1974): Die Großen Kalten Berge von Szetschuan. Zürich: Orell Füssli.

IMHOF, V. (1988): “Lebensdaten von Eduard Imhof; zusammengestellt von Viola Imhof.» In: Vermessung, Photogrammetrie, Kulturtechnik, 86. Jg. (1988), Nr. 9.

Jahresberichte 1988 der Geographischen Institute der Universität Zürich und der ETH.

KELLER, C. (1928): Lebenserinnerungen eines schweizerischen Naturforschers. Zürich und Leipzig: Orell Füssli.

KISHIMOTO, H. (1980): "Biographie Hans Boesch (1911-1978)." In: Geography and its Boundaries. A publication to commemorate the work of Prof. Dr. Hans Boesch. Bern: Kümmerly + Frey.

KYBURZ, W. (1983): "Das Geographische Institut." In: Die Universität Zürich 1933-1983. Hg. vom Rektorat der Universität Zürich, Gesamtred. Peter Stadler. Zürich: Universität Zürich.

MILT, B. (1949): Die Entwicklung der Zürcher Naturwissenschaften und ihr Aufschwung durch den Geist von 1848. Zürich: Fretz.

MÜNZER, V. und GERBER, P. R. (1989): 100 Jahre Völkerkundemuseum (1889-1989). In: [Münzer, V. et al.:] 100 Jahre Völkerkundemuseum der Universität Zürich. Zürich: Völkerkundemuseum.

Neue Zürcher Zeitung (div. Jahrgänge und Nummern).

Protokolle der Ethnographischen Gesellschaft Zürich (1888-1899) und der Geographisch-Ethnographischen Gesellschaft Zürich (1899-1989).

SCHMUTZ, H.-K. (1983): "Die Gründung des Zürcher Lehrstuhles für Anthropologie." In: Gesnerus, Bd. 40 (1983).

SPIESS, E. (1978): «Prof. Dr. Dr. h. c. Hans Boesch (24. März 1911 bis 16. August 1978)." In: Geographica Helvetica, 33. Jg. (1978), Nr. 4.

Staatsarchiv des Kantons Zürich, Dossiers U 110b. 1, U 110d, U 118b, U 1108 .

STAUB, W. und HINDERBERGER, A. (Hg.) (1943-44): Die Schweiz und die Forschung. 2 Bde. Bern: Hans Huber.

STEINER, D. (1987): «Rückgriff auf Ganzheitliches bei Ernst Winkler (11.4.1907-28.2.1987)." In: Geographica Helvetica, 42. Jg. (1987), Nr. 1.

STEINMANN, A. (1943): "Die Sammlung für Völkerkunde der Universität Zürich.» In: Mitteilungen der Geographisch-Ethnographischen Gesellschaft Zürich, Bd. 41 (1941/43).

STEINMANN, A. (1946): "Der Anteil Zürichs an der völkerkundlichen Forschung." In: Festschrift zur 200-Jahr-Feier der Naturforschenden Gesellschaft in Zürich. Zürich: Fretz.

STOLL, O. (1918): "Die Entwicklung der Völkerkunde von ihren Anfängen bis in die Neuzeit. " In: Mitteilungen der Geographisch-Ethnographischen Gesellschaft Zürich, Bd. XVIII (1917/18). 
STROHL, J. (1924): “Otto Stoll 1849-1922.» In: Vierteljahresschrift der Naturforschenden Gesellschaft in Zürich, 69. Jg. (1924)

Vorlesungsverzeichnisse der Universität Zürich 1833 ff. und der Eidgenössischen Technischen Hochschule $1855 \mathrm{ff}$.

WEHRLI, H. (1925): “Otto Stoll (1849-1922).» In: Mitteilungen der Geographisch-Ethnographischen Gesellschaft Zürich, Bd. XXIII (1922/23).

WEHRLI, H. (1939): “Zur Geschichte der Geographisch-Ethnographischen Gesellschaft [Zürich] 1888-1938". In: Mitteilungen der Geographisch-Ethnographischen Gesellschaft Zürich, Bd. XXXIX (1938/39).

WINKLER, E. (1936): "Zur Geschichte der Geographie.» In: Neue Zürcher Zeitung, 7. Januar 1936 (Nr. 31).

WINKLER, E. (1941): "Prof. Dr. Otto Lehmann, E.T.H. (1884-1941)." In: Mitteilungen der Geographisch-Ethnographischen Gesellschaft Zürich, Bd. XXXX (1939/40 und 1940/ 41).

WINKLER, E. (1977): Der Geograph und die Landschaft Festschrift zum 70. Geburtstag von Prof. Dr. E. Winkler, zusammengestellt von Gabriela Winkler und Erich Bugmann. Zürich: Atlantis.

\section{Abbildungsnachweis}

Archiv der Universität Zürich: Abb. 6, 18

Baugeschichtliches Archiv der Stadt Zürich: Abb. 5 Graphische Sammlung der Zentralbibliothek Zürich: Abb. 1

Photoswissair: Abb. 11

Vermessungsamt der Stadt Zürich: Abb. 10

Wissenschaftshistorische Sammlung der Eidgenössischen Technischen Hochschule: Abb. 2, 4, 13 a+b, 14, 18

Frau V. Imhof, Erlenbach: Abb. 7, 8, 9

Frau Prof. Dr. H. Kishimoto, Zürich: Abb. 12

Frau B. Zuidema, Zürich: Abb. 16

Herr Prof. A. Dürst, Zürich: Abb. 18

Herr A. Illi, Herrliberg: Abb. 17

Abb. 18c (Emil Egli) gezeichnet von Theo Wismann 